\title{
Extracellular vesicle-mediated cellular crosstalk in lung repair, remodelling and regeneration
}

\author{
Tsukasa Kadota $^{1,2}$, Yu Fujita ${ }^{1,2}$, Jun Araya ${ }^{1}$, Takahiro Ochiya ${ }^{3}$ and Kazuyoshi Kuwano ${ }^{1}$ \\ ${ }^{1}$ Division of Respiratory Diseases, Dept of Internal Medicine, The Jikei University School of Medicine, Tokyo, Japan. ${ }^{2}$ Dept of \\ Translational Research for Exosomes, The Jikei University School of Medicine, Tokyo, Japan. ${ }^{3}$ Institute of Medical Science, Tokyo \\ Medical University, Tokyo, Japan.
}

Corresponding author: Yu Fujita (yuugot@jikei.ac.jp)

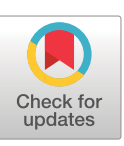

Copyright @The authors 2022

This version is distributed under the terms of the Creative Commons Attribution Non-Commercial Licence 4.0. For commercial reproduction rights and permissions contact permissions@ersnet.org

Received: 21 April 2021 Accepted: 8 Oct 2021

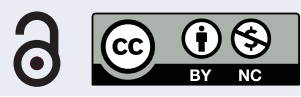

Shareable abstract (@ERSpublications)

Extracellular vesicles are important intracellular mediators in lung repair, remodelling and regeneration, and are potential therapeutic agents for lung regenerative medicine https://bit.ly/3jhc7sb

Cite this article as: Kadota T, Fujita Y, Araya J, et al. Extracellular vesicle-mediated cellular crosstalk in lung repair, remodelling and regeneration. Eur Respir Rev 2022; 31: 210106 [DOI: 10.1183/ 16000617.0106-2021].

\section{Abstract}

The unperturbed lung is highly quiescent, with a remarkably low level of cell turnover. However, once damaged, the lung shows an extensive regenerative capacity, with resident progenitor cell populations reentering the cell cycle and differentiating to promote repair. This quick and dramatic repair response requires interactions among more than 40 different cell lineages in the lung, and defects in any of these processes can lead to various lung pathologies. Understanding the mechanisms of interaction in lung injury, repair and regeneration thus has considerable practical and therapeutic implications. Moreover, therapeutic strategies for replacing lung progenitor cells and their progeny through cell therapy have gained increasing attention. In the last decade, extracellular vesicles (EVs), including exosomes, have been recognised as paracrine mediators through the transfer of biological cargo. Recent work has revealed that EVs are involved in lung homeostasis and diseases. In addition, EVs derived from specific cells or tissues have proven to be a promising cell-free modality for the treatment of lung diseases. This review highlights the EV-mediated cellular crosstalk that regulates lung homeostasis and discusses the potential of EV therapeutics for lung regenerative medicine.

\section{Introduction}

The lung is a structurally complex organ with a large and highly vascularised epithelial surface area, comprising more than 40 different cell lineages from the trachea to the alveolar spaces [1, 2]. Two major compartments are present in the lungs: the conducting airways, including the trachea, bronchi and bronchioles; and the respiratory airways, including the alveoli [3]. The conducting airways represent the first line of defence and first domain of contact between the external environment and the respiratory system, while the alveoli represent the site of gas exchange between inhaled air and the pulmonary circulation. The lung is continuously exposed to various environmental substances, including dust, smoke and pathogens, which can cause infection and injury. Under normal conditions, the lung is a highly quiescent tissue with a remarkably slow cellular turnover, compared with other organs such as the skin and gastrointestinal tract [4]. However, the lung demonstrates a significant capacity for regeneration and repair following injury. This low cellular turnover under homeostatic conditions and facultative regenerative response after injury require cell-intrinsic factors and dynamic cellular interactions between stem cells and the surrounding environment - the niche in which they reside [5]. Such stem cells receive and respond to various feedback signals originating from the stem cells themselves, neighbouring cells within the same niche, or even other tissues. Other forms of signalling such as contact-dependent Notch signalling, signalling guided by mechanical and physical cues, and signalling from the extracellular matrix through adhesion receptors also provide important signals to stem cells [6, 7]. If the regulatory process is not completed successfully, lung function can be reduced concomitant with chronic inflammation and 
pathological remodelling [8]. Understanding the crosstalk between stem cells and surrounding cells in the lung has considerable practical and therapeutic implications.

In the last decade, extracellular vesicles (EVs) including exosomes have been recognised as a new paracrine mediator through the transfer of biological cargos [9, 10]. In addition, EVs derived from specific cells or tissues present a promising cell-free modality for the treatment of lung diseases. In this review, we highlight the roles of EVs derived from each cell type in lung repair, remodelling and regeneration and their therapeutic potential for lung regenerative medicine.

Extracellular vesicles

All cells release not only soluble factors such as cytokines and growth factors, but also EVs into their environment. EVs contain various molecules such as proteins, RNA transcripts, microRNAs and active lipids, enclosed in a phospholipid bilayer derived from either the plasma membrane or endocytic compartments of the cell $[10,11]$. These enclosed molecules can be transferred to other cells, triggering a broad range of cellular activities and biological responses [9, 11, 12]. EVs are often categorised as exosomes, microvesicles (MVs) or apoptotic bodies, based on their size, biogenesis and secretory mechanisms [10]. Exosomes are approximately $100 \mathrm{~nm}$ in diameter and are generated by the fusion of multivesicular bodies (MVBs) with the plasma membrane. Fusion of the MVB with the plasma membrane is in part regulated by neutral sphingomyelinase 2, endosomal sorting complex required for transport, tetraspanins, Rab proteins, syntenin, programmed cell death 6 interacting protein and phospholipase D2 [13-15]. In contrast, MVs are a few nanometres to a few micrometres in diameter and are generated by outward budding from the plasma membrane. These EVs are rich in several lipids and phosphatidylserine and contain membrane components similar to those of the parental cell membrane [16, 17]. Apoptotic bodies are several micrometres in diameter and are released from the plasma membrane during cell apoptosis via indiscriminate blebbing [18]. However, current methods cannot distinguish between these different types of EVs because of overlapping physical characteristics [9, 10]. For instance, the small MVs, exosomes and enveloped viruses share the same biophysical characteristics in terms of size, density and membrane orientation [9]. Most studies therefore rely on regular EV isolation techniques that, even if they use the term "exosomes", analyse very heterogeneous mixtures of different EVs. Based on the evidence, the International Society for Extracellular Vesicles consensus recommends using the generic term EV for particles naturally released from the cell in the nomenclature [10].

EVs are involved in various cell-to-cell signalling pathways and act as important molecular messengers in diverse biological and pathological processes [12, 19]. As a result, investigations into EV-mediated intercommunication have elucidated other important mechanisms behind normal homeostasis and diseases. More details on EV-mediated intercommunication mechanisms and various EV-specific functions can be found in several recent reviews [9, 10, 13, 19-22]. Importantly, EVs appear to play diverse roles in the subsequent behaviours of various stem/progenitor cells and cells within their niche in a region-dependent manner during regeneration.

EV-mediated lung microenvironment at homeostasis and in response to injury

Different regions of the human lung exhibit different strategies for lung regeneration. The human proximal airways start with the trachea, which then branches to give rise to bronchi, each of which then gives rise to bronchioles, which eventually terminate in tiny air sacs called alveoli. In the proximal region, the airway is lined by a pseudostratified epithelium of basal cells, ciliated cells and secretory cells (club and goblet cells), as well as small numbers of neuroendocrine cells, ionocytes and tuft cells [2, 23-26]. Basal cells act as major progenitor cells that self-renew and, when necessary, give rise to multiple cell types such as secretory, goblet, ciliated and neuroendocrine cells to maintain the epithelial integrity of the proximal airways [27-29]. In addition to basal cells, certain populations of secretory cells can possess both the ability to self-renew and to transform into differentiated cell types for purposes of replacement. In the distal region, alveoli are distinct in structure, comprising tiny air sacs composed of alveolar type 1 (AT1) and alveolar type 1 (AT2) cells. It is well accepted that AT2 cells can act as stem/progenitor cells in the alveolar epithelium during development and injury in the adult lung [24, 30, 31]. Furthermore, there is emerging evidence of the importance of the stromal cells that support the epithelial stem cell niche, including mesenchymal and immune cells in lung regeneration. Here we describe the EV-mediated signals from lung stem/progenitor cells themselves and the neighbouring cells (figure 1).

\section{Airway epithelial cell-derived EVs}

Basal cells are cuboidal in shape, secured to the basement membrane and are specifically characterised by the expression of transformation-related protein 63 (Trp63), cytokeratin 5 (Krt5) and nerve growth factor receptor [23]. Basal cells in the mouse lung continuously self-renew at a low rate and the homeostatic 
Normal adult lung

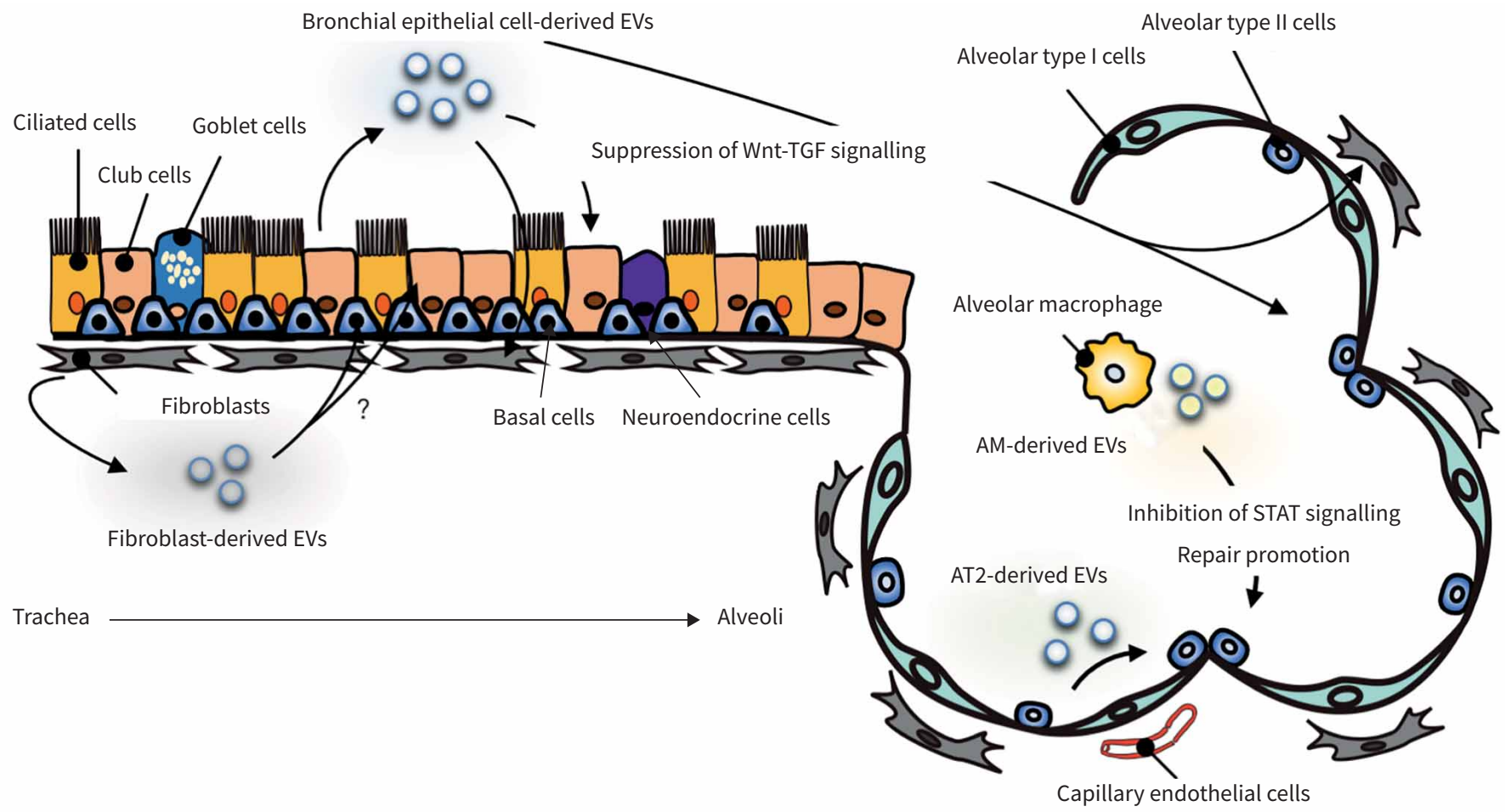

FIGURE 1 Roles of extracellular vesicles (EVs) in lung normal and injured stem cell niches. We propose that EVs derived from epithelial stem cells and the surrounding cells maintain lung quiescence under conditions of lung homeostasis and regulate repair and regeneration after injury. Human bronchial epithelial cell-derived EVs inhibit transforming growth factor- $\beta$ (TGF- $\beta$ )-mediated induction of epithelial cell senescence and myofibroblast by attenuating Wnt signalling in the lung. Alveolar macrophage-derived EVs mediate inhibition of signal transducer and activator of transcription (STAT) activation in epithelial cells by delivering vesicular suppressor of cytokine signalling (SOCS) proteins after injury. Alveolar macrophage-derived EVs also promote proliferation of lung epithelial cells via shuttling miR-221/222 after injury. AM: alveolar macrophage; AT1: alveolar type 1; AT2: alveolar type 2.

turnover is regulated in part via fibroblast growth factor (FGF) receptor 2 and bone morphogenetic protein (BMP) [32, 33]. Notch signalling regulates their preferable differentiation towards the secretory cell lineage in homeostasis and repair [29, 34, 35]. In addition, other signalling types are known to impact cell proliferation and growth in murine injury repair models, including Wnt signalling [36-38], Hippo signalling [39] and p53 [40]. Moreover, basal cells can also proliferate and migrate to the alveolar regions of the lung after severe injury [41]. These basal-like cells also express Trp63 and Krt5, and have been seen to contribute to injury repair [42, 43]. In these cells, the Notch pathway is activated for repair after injury, which can induce differentiation of basal cells into secretory cells [29]. In recent years, signals between stem cells and their daughter cells and stem cell self-signalling loops in homeostasis and repair have been a focus of attention [34, 44]. However, the mechanisms underlying quiescence and response to injury in the lung have yet to be fully elucidated. In particular, interactions between airway epithelial cells and alveolar epithelial cells remain poorly understood.

EVs derived from airway epithelial cells can be involved in epithelial cell homeostasis in airways and alveoli. GuPTA et al. [45] reported that airway epithelial cells can communicate with each other through EVs. Treatment with EVs derived from different types of human airway cells resulted in differential expression/regulation of certain proteins and microRNAs (miRNAs) in the target airway epithelial cells. Our recent study highlighted the finding that EVs derived from human bronchial epithelial cells, which express Trp63 and Krt5, can be transferred to epithelial cells and fibroblasts in airway and alveoli, and may inhibit epithelial cell senescence and myofibroblast differentiation via inhibition of transforming growth factor- $\beta$ (TGF- $\beta$ ) and Wnt signalling pathways [46] (figure 1). Mechanistically, the EV miRNA cargo is primarily responsible for attenuating both myofibroblast differentiation and cellular senescence by inhibition of Wnt3A, Wnt5A and Wnt10B. Crosstalk between the TGF- $\beta$ superfamily and Wnt signalling 


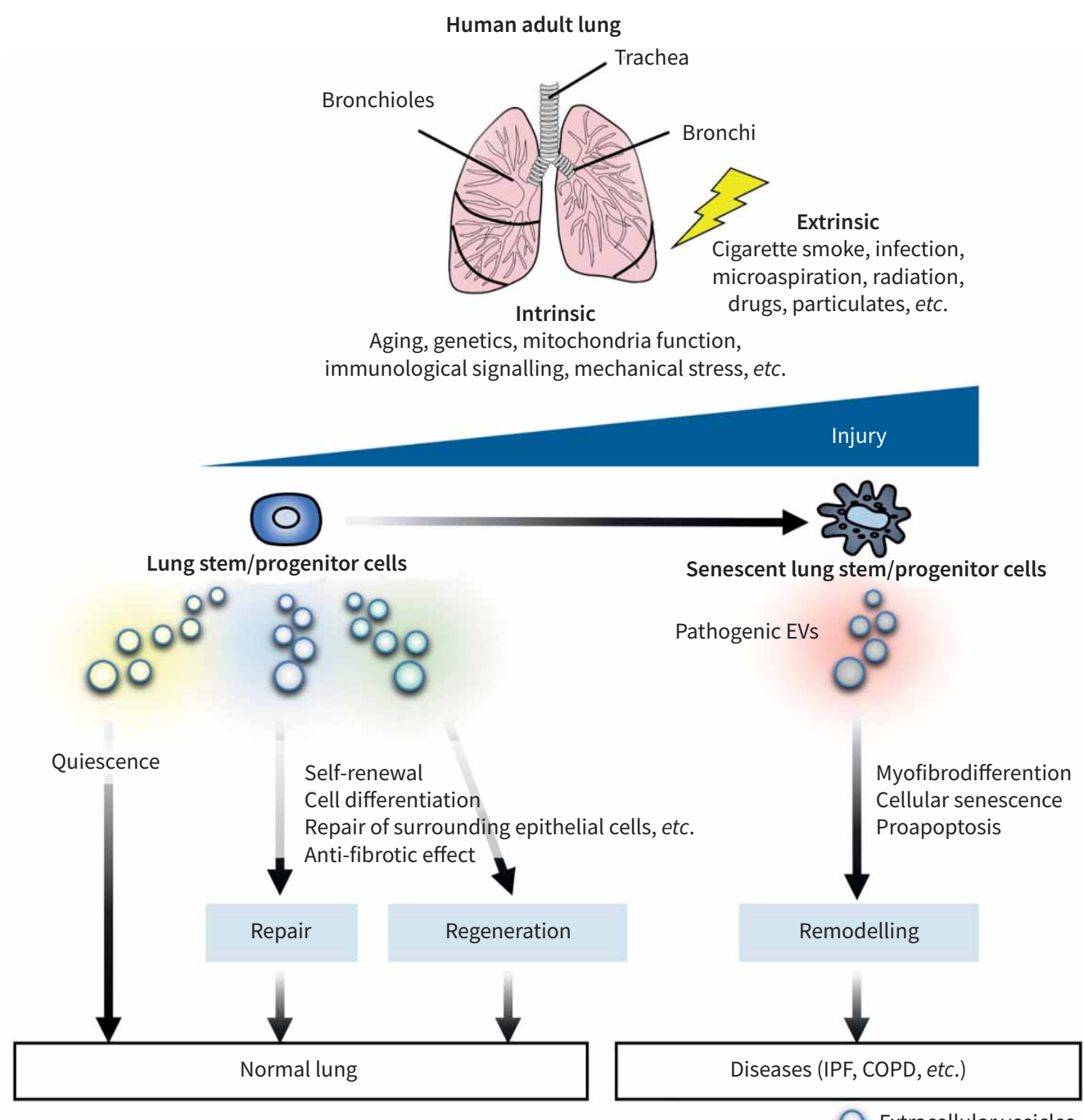

FIGURE 2 Proposed model of the involvement of epithelial cell-derived extracellular vesicles (EVs) in injury-repair responses in the lung. Intrinsic (e.g., ageing, genetics, mitochondria function, immunological signalling, mechanical stress) and extrinsic (e.g., cigarette smoke, infection, microaspiration, radiation, drug) sources of injury can cause epithelial cell death or cellular senescence, leading to dysfunction of resident epithelial stem cells. In the absence of injury, epithelial stem cell-derived EVs maintain lung quiescence. Under injury, epithelial stem cell-derived EVs can preserve lung homeostasis (repair). However, if these are unsuccessful, the EVs can also help to regenerate lung tissue. On the contrary, pathogenic EVs derived from damaged epithelial stem cells (e.g., senescent cells) develop a remodelling, which can be involved in lung pathogenesis such as idiopathic pulmonary fibrosis (IPF) and chronic obstructive pulmonary disease (COPD).

pathways plays an essential role in regulating stem/progenitor cell and mesenchyme functions during quiescence and injury repair $[47,48]$. In alveoli, canonical Wnt signalling is activated after injury, while the activity is low in most epithelial cells, including AT2 cells [49]. During alveoli regeneration after injury, active Wnt signalling is essential for the expansion of a Wnt-responsive subpopulation of AT2 cells and the inhibition of differentiation of AT2 to AT1 cells [50]. In airways, basal cells also activate canonical Wnt signalling following tracheal damage [36-38]. Thus, through regulation of TGF- $\beta$ and Wnt signalling, bronchial epithelial cell-derived EVs may maintain quiescent state and self-renewal, and regulate their repair and regeneration in alveolar and airway (figure 2).

\section{Alveolar epithelial cell-derived EVs}

The alveoli are distinct in structure, comprising tiny air sacs composed of AT1 and AT2 cells. Alveolar epithelial cells show a relatively slow rate of cellular turnover under normal conditions. However, after 
injuries such as diphtheria toxin-mediated AT2-specific ablation, pneumonectomy, or hyperoxia-induced injury [51-53], AT2 cells show a robust regenerative capacity through extensive cell proliferation and differentiation into AT1 cells in mice. Recent studies have shown several distinct subclassifications of AT2 cells according to single-cell transcriptomic data. For example, two clusters of AT2 cells have been identified in the human lung [2]. One cluster expressed higher levels of some canonical AT2 markers such as surfactant protein C, and other selectively expressed genes involved in Wnt signalling and detoxification. In addition, in response to acute injury, a Wnt-responsive subpopulation of AT2 cells has been identified as major facultative progenitor cells in the distal lung [50, 54]. Currently, in addition to Wnts and their antagonists, a number of other signalling factors such as BMPs, endothelial growth factor (EGF) and vascular EGFs (VEGFs) have been identified as regulators within the alveolar stem cell niche $[55,56]$. Inflammatory conditions are also required for epithelial maintenance [57]. However, the cellular and molecular mechanisms underlying alveolar niche construction remain largely unknown.

AT2 cells can communicate with each other for the maintenance or repair of injured alveoli through EVs (figure 1). QuAN et al. [58] showed that human AT2 cell-derived EVs support AT2 cell-specific proliferation via miR-371b-5p transfer in response to lung injury. They showed that miR-371b-5p in EVs derived from the AT2 cell line (A549) promoted AT2 cell-specific proliferation, but not differentiation in pluripotent stem cell-derived cultures. They also observed that bleomycin-treated human-induced pluripotent stem cell-differentiated AT2 cells (hiPSC-AT2) and primary human AT2 cells secrete miR-371b-5p in EVs. These results suggest the possibility that AT2 cell-derived EV miR-371b-5p may serve as alveolar niche signalling in response to lung injury, promoting alveolar re-epithelialisation [58]. Mitchell et al. [59] suggested the potential beneficial effects of human AT2 cell-derived EVs on hyperoxia-induced lung injury in a mouse model. They administered EVs derived from hiPSCs and hiPSC-AT2 to mice with hyperoxia-induced lung injury and found that those mice receiving the EVs displayed less damage than mice receiving the control EV. These results imply that AT2 cell-derived EVs may promote repair of surrounding epithelial cells after injury in alveoli (figure 1). Currently, the roles of EVs derived from AT1 cells and subsets of AT2 cells such as the Wnt-responsive subpopulation remain unknown in both normal alveolar homeostasis and response to injury.

\section{Mesenchymal cell-derived EVs}

The mesenchymal niche surrounding the airways and alveoli plays essential roles in both maintaining homeostasis and regenerating epithelium. The mesenchyme displays anatomically distinct cell types and region-dependent heterogeneity [60, 61]. These populations include myofibroblasts, lipofibroblasts, adventitial fibroblasts, alveolar fibroblasts and fibromyocytes [2, 62, 63]. In the trachea, mesenchymal lineages promote epithelial repair [60, 62]. Recent work has shown that airway mesenchyme including smooth muscle, which expresses important factors such as FGF10, acts in a paracrine fashion to support the regeneration of basal cells in mice [61, 64]. FGF10 is a potent pro-regenerative growth factor that poses protective and therapeutic effects in the context of various injury models [65]. Evidence indicates that most leucine-rich repeat-containing G-protein coupled receptor 6 (Lgr6)-expressing cells are airway smooth muscle cells, which promote airway differentiation of epithelial progenitors via Wnt-FGF10 crosstalk in response to naphthalene injury [60]. In the alveoli, lung fibroblasts (LFs) and AT2 cells have direct and extensive contacts [66]. These alveolar interstitial fibroblasts, especially platelet-derived growth factor receptor alpha (PDGFR $\alpha$ )-expressing fibroblasts (including lipofibroblasts), have a critical role to play in homeostatic alveolar regeneration following injury [51, 62, 67]. Moreover, Lgr5-expressing cells are located in alveolar compartments and appear to be distinct from PDGFRo-expressing cells. This population is sufficient to promote alveolar differentiation of epithelial progenitors through Wnt activation [60]. Furthermore, mesenchymal cells have been suggested to promote differentiation of AT1 cells and suppress AT2 cell proliferation via BMP antagonist production [56, 62]. Although considerable research efforts have been undertaken to elucidate mesenchymal-to-epithelial crosstalk, the mechanisms and pathways are not fully understood.

To date, no direct evidence has elucidated the EV-mediated mesenchymal feedback to epithelial stem/ progenitor cells during regeneration. However, EVs derived from LFs isolated from the lung tissue of patients can drive abnormal epithelial regeneration. For example, in the airways, HaJ-SALEm et al. [68] showed that bronchial fibroblasts derived from severe eosinophilic asthma patients promote epithelial cell proliferation by delivering lower levels of TGF- $\beta 2$, which may be involved in an established feature of airway remodelling. Conversely, in alveoli, we have reported that LFs from patients with idiopathic pulmonary fibrosis (IPF) have myofibroblast and senescent phenotypes and secrete EVs, which induce mitochondrial damage and subsequent cellular senescence in lung epithelial cells [69]. Together with the need for co-culture of primary AT2 cells with PDGFR $\alpha$-positive fibroblasts [70] and the beneficial effects of the secretome from mesenchyme on pathologic alveolar epithelial cells [71], these observations suggest 
promising roles of LF-derived EVs in epithelial stem cell homeostasis and response to injury (figure 1). Furthermore, EVs from stimulated LFs affect neighbouring fibroblasts in the lung [72]. Interleukin (IL)-1 $\beta$-treated fibroblasts produce antifibrotic prostaglandin-containing EVs that can inhibit TGF- $\beta 1$-induced myofibroblast differentiation of naïve LFs [72]. Overall, further work is needed to define the functional roles of EVs derived from heterogeneous populations of mesenchymal cells in lung homeostasis and regeneration.

\section{Immune cell-derived EVs}

The lung contains various populations of resident immune cells with multiple subclasses of dendritic cells, innate lymphoid cells and interstitial and alveolar macrophages. After acute lung injury, immune cells are recruited to the alveolar region and can promote alveolar regeneration by modulating the regenerative niche $[73,74]$. Macrophages and monocytes appear to play distinct roles in this process. Resident alveolar macrophages can directly support the growth of AT2 cell proliferation and self-renewal [75, 76]. Recent studies have shown that bone marrow-derived macrophages are recruited in the lung through a $\mathrm{C}-\mathrm{C}$ motif ligand 2-C-C chemokine receptor 2 axis after partial pneumonectomy and are involved in the compensatory regrowth of AT2 cells [75]. To date, various cytokines and growth factors secreted by immune cells, such as IL- $1 \beta$, TNF $\alpha$ and IL-6, have been shown to exert direct effects on the airway or alveolar niche [76-78].

Macrophages are thought to be significant producers of EVs in the lung [22, 79]. EVs derived from macrophages can promote epithelial regeneration by modulating the regenerative niche (figure 1). For example, human alveolar macrophages (AMs) secrete suppressor of cytokine signalling (SOCS) 1 and SOCS3 proteins in EVs that can be taken up by alveolar epithelial cells to mediate inhibition of signal transducer and activator of transcription (STAT) activation in response to cytokines, such as IL-6 [80]. Janus kinase-STAT signalling is critical in transducing the effects of many cytokines, hormones and growth factors. A family of STAT-induced STAT inhibitors termed SOCS proteins act in a classical negative-feedback loop to inhibit cytokine signal transduction [81]. In addition, secretion of SOCS3 within EVs derived from resident human AMs can inhibit lung tumourigenesis and dampen allergic airway inflammation by inhibiting the production of type 2 immune response-associated cytokines such as IL-4, IL-13 and thymic stromal lymphopoietin from airway epithelial cells [82]. Furthermore, after stimulation by lipopolysaccharide (LPS), macrophage-derived EVs, particularly apoptotic bodies, can promote proliferation of lung epithelial cells via shuttling miR-221/222 [83]. Moreover, antifibrotic properties are enriched in EVs from the sputum and plasma of patients with IPF, and these EVs are suggestive of a macrophage origin [84]. These studies highlight that macrophage-derived EVs are crucial in the maintenance of epithelial cells (figure 1).

\section{Lung-resident mesenchymal stem/stromal cell-derived EVs}

MSCs are a unique subset of progenitor cells defined by their capacity to differentiate into multiple mesenchymal lineages such as bone, cartilage, muscle and adipose tissue [85]. They are typically found in a variety of human tissue, including bone marrow, adipose tissue, umbilical cords, placenta, lung, and other tissues. Lung-resident MSCs reside in the perivascular niche and can be activated and recruited to the site of injury, which contributes to the maintenance of lung integrity. Emerging evidence indicates that they can orchestrate immunomodulation, lung repair and regeneration [86]. Although the mechanisms of action of these MSCs in lung injuries and diseases have not yet been fully elucidated, the beneficial effects are at least partially dependent on paracrine mechanisms including release of bioactive molecules and EVs [87, 88].

Lung-resident MSC-derived EVs may be involved in repair and regeneration of lung tissue. EVs derived from MSCs from different sources have been shown to be beneficial to experimental viral, bacterial and LPS-induced lung injury models [89-92]. SiLva et al. [93] showed that MSC-derived EVs modulate alveolar-capillary barrier integrity through mitochondrial transfer. It has been reported that MSC-derived EVs contain mitochondria [94]. The mitochondrial transfer via EVs could attenuate mitochondrial dysfunction and restore mitochondrial respiration impaired by LPS stimulation in primary human distal lung epithelial cells. YI et al. [95] found that miR-30b-3p in MSC-derived EVs is transferred to human alveolar epithelial cells, which inhibit expression of serum amyloid A3 (SAA3). SAA3 is a chief member of the SAA family and a major component of the acute phase of inflammation. EVs derived from miR-30b-3p-overexpressing MSCs resulted in an anti-inflammatory and pro-reparative effect in LPS-treated mouse alveolar epithelial cells through inhibition of SAA3. Li et al. [96] showed that MSCs could ameliorate ischaemia/reperfusion lung injury via EV secretion. MSC-derived EV treatment could reduce lung oedema, pulmonary dysfunction and inflammation that were associated with ischaemia/reperfusion injury in mice. It could also reduce hypoxia/reoxygenation-induced lung epithelial cell apoptosis. KHATRI 
et al. [89] investigated the effect of MSC-derived EVs in influenza virus-induced acute respiratory distress syndrome in a pig model. In the study, MSC-derived EVs inhibited the replication of influenza virus and the virus-induced apoptosis in human lung epithelial cells. WeI et al. [97] demonstrated that miR-21-5p in MSC-derived EVs reduced oxidative stress-induced apoptosis in mouse lung epithelial cells. Although there is limited evidence of lung-resident MSC-derived EVs in the stem cell niche, these results support the notion that they exert protective effects against lung epithelial injury. Further studies are needed to understand their importance in the context of homeostasis and disease of lung, since lung-resident MSC-derived EVs could be instrumental in providing more specific and targeted therapies for lung diseases.

\section{EV-mediated tissue remodelling in pulmonary fibrosis and COPD}

Lung injury can induce a dysplastic repair response in the epithelium and mesenchyme. Irreversible lung remodelling by chronic aberrant injury and inflammation is considered a primary driver of lung diseases such as pulmonary fibrosis and COPD. EV cargos have been proved to reflect cell types and their physiological and pathological conditions of donor cells [98]. Although EVs secreted from stem/progenitor cells exhibit beneficial effects on lung repair and regeneration, EVs from other cell types such as senescent cells and activated neutrophils can lead to tissue remodelling in the lung (figure 2).

IPF is the most common form of fibrotic pulmonary disease, representing a progressive, irreversible and fatal lung disease characterised by diffuse alveolar epithelial cell injury and structural remodelling. Although the aetiology has not been clearly delineated, continuing injury to the epithelium, aberrant activation of the epithelium, impaired epithelial regenerative capacity, and fibroproliferative responses in the ageing lung are well-accepted theories for the pathogenesis of IPF [99]. Several recent studies have highlighted the roles of EVs on the epithelial phenotype and fibroproliferative response in IPF pathogenesis. Our recent study showed that LF-derived EVs from IPF patients increase mitochondrial reactive oxygen species (mtROS) and associated mitochondrial damage to lung epithelial cells, leading to mtROS-mediated activation of DNA damage responses and subsequent epithelial cell senescence [69]. Another group showed that Wnt5A secretion is increased in EVs from bronchoalveolar lavage fluid among patients with IPF, promoting the proliferation of LFs mediated by Wnt5A [100]. In addition, fibroblasts undergoing replicative senescence or TGF- $\beta 1$-induced senescence promote fibroblast invasion, which is caused by direct interaction of fibronectin localised to the EV surface with recipient fibroblasts [101].

COPD is characterised by chronic, progressive and irreversible airway limitations and is caused by the inhalation of cigarette smoke and other noxious particles. The pathologic hallmarks of COPD are characterised by the emphysematous destruction of the alveolar structure and the remodelling and narrowing of small airways. Our study showed that cigarette smoke extract (CSE) triggers the modification of EV components of bronchial epithelial cell-derived EVs, which promoted the differentiation of LFs into myofibroblasts [102]. Mechanistically, an elevated level of miR-210 in CSE-stimulated bronchial epithelial cell can regulate autophagy via targeting ATG7 in LFs, leading to myofibroblast differentiation. Furthermore, GENSChmER et al. [103] revealed the role of neutrophil-derived EVs in the pathogenesis of COPD via promoting proteolytic damage. Activated neutrophil-derived EVs could bind and degrade extracellular matrix via the integrin Mac-1 and neutrophil elastase, causing the hallmarks of COPD. SONI et al. [104] also showed that the bronchoalveolar lavage fluid levels of neutrophil-derived EVs correlated with the disease severity of patients with COPD. Such findings provide evidence that the disruption of proper EV secretion or secretion of pathogenic EVs after injury results in inefficient lung repair and regeneration, and subsequent remodelling of the lung.

\section{EV therapy for lung regeneration}

Stem cell-based therapy as a modality of regenerative medicine is considered an attractive treatment option for various lung diseases [105-108]. The therapeutic effects of stem cells can be attributed to three main mechanisms of action: homing, that is, migration to the site of injury; differentiation into various cell types that can repair the damaged tissue; and secretion of bioactive factors [109]. Among these mechanisms, recent studies suggest that stem cell paracrine effects are primarily responsible for the regenerative potential $[110,111]$ and may be advantageous given the risks associated with stem cell use, such as immune compatibility, tumorigenicity, and transmission of infections [112]. Cell-free secretome-based therapies, including the use of EVs, have thus been proposed as a potentially efficacious alternative to cell injection approaches. EVs secreted from stem cells are naturally loaded with various bioactive molecules derived from stem cells and offer several advantages for clinical application. First, the smaller size of EVs is suitable for delivery and deposition in the small airways and alveolar regions responsible for many respiratory diseases via the modality of inhalation therapy. Second, EVs are stable in tissues and body fluids due to their lipid bilayer architecture. Third, EVs exhibit low levels of immunogenicity and toxicity 
compared to cell therapies. To date, various types of stem cell-derived EVs have been studied for respiratory diseases in experimental models and in clinical trials, such as MSCs [91, 92, 113], human bronchial epithelial cells [46], lung tissue spheroid cells [114], endothelial progenitor cells and induced pluripotent stem cells [59].

MSC-derived EVs have various effects such as immunosuppression and regeneration. Numerous preclinical and clinical studies have suggested that MSC-derived EVs hold promise as therapeutics for respiratory diseases such as COPD, IPF and acute respiratory distress syndrome [88, 91, 92, 113, 116]. The mechanisms of action and clinical applications of MSC-derived EVs for respiratory diseases have been the topic of several comprehensive reviews, including ours [88, 116].

Lung-resident airway epithelial cell-derived EVs have therapeutic effects for IPF [46]. Human bronchial and small airway epithelial cell-derived EVs can suppress TGF- $\beta$-induced myofibroblast differentiation and lung epithelial cell senescence, and the effects are more potent than those observed with MSC-EVs. Mechanistically, the bronchial epithelial cell-derived EV miRNA cargo is primarily responsible for attenuating both myofibroblast differentiation and cellular senescence. This attenuation occurs via inhibition of canonical and non-canonical Wnt signalling pathways. Mouse models utilising intratracheal administration of EVs demonstrate efficient attenuation of bleomycin-induced lung fibrosis development accompanied by reduced expression of both $\beta$-catenin and markers of cellular senescence [46].

Lung tissue-derived EVs have therapeutic effects on their native tissues. EVs derived from lung spheroid cells, which contain a heterogeneous population of cells expressing lung epithelial and mesenchymal markers, attenuate and resolve bleomycin- and silica-induced fibrosis by re-establishing normal alveolar structure and decreasing both collagen accumulation and myofibroblast proliferation [114]. Although the mechanisms were not fully elucidated in that study, anti-fibrotic miRNAs such as miR-30a and the let-7 and miR-99 family in EVs may be partially involved in the attenuation of fibrosis.

Endothelial progenitor cells (EPCs) are involved in several lung diseases, such as COPD, pulmonary hypertension and bronchopulmonary dysplasia. Systemic administration of EPCs can partially improve alveolar and endothelial damage [117]. These therapeutic effects are caused, at least partially, by paracrine effects. EVs from human EPCs isolated from the cord blood from a healthy pregnant woman reduced lung injury in LPS-induced acute lung injury mice. This effect was partly achieved by delivering the miRNA-126 in EVs into the injured epithelial cells, thus modulating the expression of a number of relevant genes, including cytokines, VEGF $\alpha$ and tight junction components [115].

HiPSCs are derived from adult somatic cells and are reprogrammed into a pluripotent state by induced expression of certain transcription factors, such as Oct4, Sox2, Myc and Klf4 [118, 119]. Induced pluripotent stem cell (iPSC) secretome, including EVs has been reported to improve hyperoxia-induced acute lung injury, bleomycin-induced fibrosis, and postpneumonectomy lung structure and function [59, 120-122]. However, clear evidence for the efficacy of iPSC-derived EVs in improving lung diseases remain elusive, although one study may have shown some beneficial effects [59]. Considering the effects of the iPSC secretome, iPSC-derived EVs may have some therapeutic potential. Future studies are needed to investigate which of the MSCs or many other candidate lung cell populations, such as lung resident progenitors and iPSCs, might have the best relative potential in EV therapy to reconstitute an injured lung.

\section{Conclusions and perspectives}

Because of the inherent architectural complexity and region-dependent unique population of cell lineages, highly orchestrated cell-to-cell communications are central to the remarkable regenerative capacity of the lung after injury. Recent work suggests EV-mediated cellular crosstalk as a novel regulator of lung repair and regeneration. In particular, both airway and alveolar epithelial cell-derived EVs can play key roles in lung repair and regeneration. Furthermore, after lung injury, disruption of proper EV secretion or secretion of pathogenic EVs can lead to lung remodelling. Moreover, the containing cargo and function of EVs are dependent on not only cell type but also specific microenvironments including cigarette smoke exposure, cytokines and culturing condition. For example, normal human bronchial epithelial cell-derived EVs showed an anti-fibrotic property through suppressing myofibroblast differentiation and cellular senescence. In contrast, EVs derived from cigarette smoke-exposed human bronchial epithelial cells can induce myofibroblast differentiation associated with airway remodelling. Importantly, in addition to its roles in lung regeneration, stem cell-derived EVs have potential therapeutic functions in respiratory diseases. Thus, a better understanding of the roles of EVs derived from stem cells in the lung will facilitate the discovery and development of novel therapeutic agents for respiratory diseases. However, many questions remain to be addressed in future studies. A major challenge is how to culture lung-resident stem cells while retaining 
their phenotype. For example, the distinct pulmonary epithelial phenotype of AT2 cells is lost within few days in two-dimensional cultures. Moreover, current culture media are poorly defined and contain unknown factors derived from fetal bovine serum or bovine pituitary extracts [70]. Such complex conditions represent an obstacle to evaluating cell-type-specific EVs properly, because stem cells can differentiate into other types of cells. In addition, a further understanding of the precise characterisation of EVs remains to be solved for revealing the EV-mediated mechanisms underlying lung repair and regeneration. This limitation is due to the technical difficulty in isolating and characterising pure populations of specific subtypes of EVs. Furthermore, elimination of xenogeneic components is needed, at least during the $\mathrm{EV}$ production and harvesting phase, in order to allow use in clinical therapeutics. In recent years, a variety of in vitro culture models have been studied, such as three-dimensional organoid culture methods and large-scale mass culture systems [70]. More recently, chemically defined conditions for human AT2 cell expansion and differentiation in alveolosphere cultures have been reported [123, 124]. Future experiments using these stem cell culture methods will be crucial to reveal the EV-mediated crosstalk in lung repair and regeneration, which will facilitate the development of novel EV therapeutics.

Provenance: Submitted article, peer reviewed.

Author contributions: T. Kadota performed the literature search, wrote the manuscript, and prepared the figures. Y. Fujita, J. Araya, T. Ochiya, and K. Kuwano edited and revised the manuscript.

Conflict of interest: None declared.

Support statement: This work was partially supported by Japan Society for the Promotion of Science KAKENHI (grants: 19K17649, 21H02930) and Japan Agency for Medical Research and Development, AMED (grants: JP20fk0108284, JP21ym0126007) to Y. Fujita. Funding information for this article has been deposited with the Crossref Funder Registry.

\section{References}

1 Franks TJ, Colby TV, Travis WD, et al. Resident cellular components of the human lung: current knowledge and goals for research on cell phenotyping and function. Proc Am Thorac Soc 2008; 5: 763-766.

2 Travaglini KJ, Nabhan AN, Penland L, et al. A molecular cell atlas of the human lung from single-cell RNA sequencing. Nature 2020; 587: 619-625.

3 Tata PR, Rajagopal J. Plasticity in the lung: making and breaking cell identity. Development 2017; 144: 755-766.

4 Rock JR, Hogan BLM. Epithelial progenitor cells in lung development, maintenance, repair, and disease. Annu Rev Cell Dev Biol 2011; 27: 493-512.

5 Peng T, Frank DB, Kadzik RS, et al. Hedgehog actively maintains adult lung quiescence and regulates repair and regeneration. Nature 2015; 526: 578-582.

6 Chacón-Martínez CA, Koester J, Wickström SA. Signaling in the stem cell niche: regulating cell fate, function and plasticity. Development 2018; 145: dev165399.

7 Vining $\mathrm{KH}$, Mooney DJ. Mechanical forces direct stem cell behaviour in development and regeneration. Nat Rev Mol Cell Biol 2017; 18: 728-742.

8 Beers MF, Morrisey EE. The three R's of lung health and disease: repair, remodeling, and regeneration. $J$ Clin Invest 2011; 121: 2065-2073.

9 Mathieu M, Martin-Jaular L, Lavieu G, et al. Specificities of secretion and uptake of exosomes and other extracellular vesicles for cell-to-cell communication. Nat Cell Biol 2019; 21: 9-17.

10 Théry C, Witwer KW, Aikawa E, et al. Minimal information for studies of extracellular vesicles 2018 (MISEV2018): a position statement of the International Society for Extracellular Vesicles and update of the MISEV2014 guidelines. J Extracellular Vesicles 2018; 7: 1535750.

11 Russell AE, Sneider A, Witwer KW, et al. Biological membranes in EV biogenesis, stability, uptake, and cargo transfer: an ISEV position paper arising from the ISEV membranes and EVs workshop. J Extracell Vesicles 2019; 8: 1684862.

12 Kosaka N, Iguchi H, Yoshioka Y, et al. Secretory mechanisms and intercellular transfer of MicroRNAs in living cells. J Biol Chem 2010; 285: 17442-17452.

13 Yáñez-Mó M, Siljander PR-M, Andreu Z, et al. Biological properties of extracellular vesicles and their physiological functions. J Extracellular Vesicles 2015; 4: 27066.

14 Raposo G, Stoorvogel W. Extracellular vesicles: exosomes, microvesicles, and friends. J Cell Biol. 2013; 200: 373-383.

15 Kosaka N, Iguchi H, Hagiwara K, et al. Neutral sphingomyelinase 2 (nSMase2)-dependent exosomal transfer of angiogenic microRNAs regulate cancer cell metastasis. J Biol Chem 2013; 288: 10849-10859. 
Tkach M, Théry C. Communication by extracellular vesicles: where we are and where we need to go. Cell 2016; 164: 1226-1232.

Muralidharan-Chari V, Clancy JW, Sedgwick A, et al. Microvesicles: mediators of extracellular communication during cancer progression. J Cell Sci 2010; 123: 1603-1611.

György B, Szabó TG, Pásztói M, et al. Membrane vesicles, current state-of-the-art: emerging role of extracellular vesicles. Cell Mol Life Sci 2011; 68: 2667-2688.

Kosaka N, Yoshioka Y, Fujita Y, et al. Versatile roles of extracellular vesicles in cancer. J Clin Invest 2016; 126: 1163-1172.

Kadota T, Fujita Y, Yoshioka Y, et al. Emerging role of extracellular vesicles as a senescence-associated secretory phenotype: insights into the pathophysiology of lung diseases. Mol Aspects Med 2018; 60: 92-103.

Kadota T, Fujita Y, Yoshioka Y, et al. Extracellular vesicles in chronic obstructive pulmonary disease. Int J Mol Sci 2016; 17: 1801.

Fujita Y, Kosaka N, Araya J, et al. Extracellular vesicles in lung microenvironment and pathogenesis. Trends Mol Med 2015; 21: 533-542.

Hogan BL, Barkauskas CE, Chapman HA, et al. Repair and regeneration of the respiratory system: complexity, plasticity, and mechanisms of lung stem cell function. Cell Stem Cell 2014; 15: 123-138.

Zepp JA, Morrisey EE. Cellular crosstalk in the development and regeneration of the respiratory system. Nat Rev Mol Cell Biol 2019; 20: 551-566.

Plasschaert LW, Žilionis R, Choo-Wing R, et al. A single-cell atlas of the airway epithelium reveals the CFTR-rich pulmonary ionocyte. Nature 2018; 560: 377-381.

Montoro DT, Haber AL, Biton M, et al. A revised airway epithelial hierarchy includes CFTR-expressing ionocytes. Nature 2018; 560: 319-324.

Shivaraju M, Chitta UK, Grange RMH, et al. Airway stem cells sense hypoxia and differentiate into protective solitary neuroendocrine cells. Science 2021; 371: 52-57.

Rock JR, Onaitis MW, Rawlins EL, et al. Basal cells as stem cells of the mouse trachea and human airway epithelium. Proc Natl Acad Sci USA 2009; 106: 12771-12775.

Rock JR, Gao X, Xue Y, et al. Notch-dependent differentiation of adult airway basal stem cells. Cell Stem Cell 2011; 8: 639-648.

Basil MC, Katzen J, Engler AE, et al. The cellular and physiological basis for lung repair and regeneration: past, present, and future. Cell Stem Cell 2020; 26: 482-502.

Leach JP, Morrisey EE. Repairing the lungs one breath at a time: how dedicated or facultative are you? Genes Dev 2018; 32: 1461-1471.

Balasooriya GI, Goschorska M, Piddini E, et al. FGFR2 is required for airway basal cell self-renewal and terminal differentiation. Development 2017; 144: 1600-1606.

Tadokoro T, Gao X, Hong CC, et al. BMP signaling and cellular dynamics during regeneration of airway epithelium from basal progenitors. Development 2016; 143: 764-773.

Pardo-Saganta A, Law BM, Tata PR, et al. Injury induces direct lineage segregation of functionally distinct airway basal stem/progenitor cell subpopulations. Cell Stem Cell 2015; 16: 184-197.

Mori M, Mahoney JE, Stupnikov MR, et al. Notch3-Jagged signaling controls the pool of undifferentiated airway progenitors. Development 2015; 142: 258-267.

Lynch TJ, Anderson PJ, Xie W, et al. Wnt signaling regulates airway epithelial stem cells in adult murine submucosal glands. Stem Cells 2016; 34: 2758-2771.

Brechbuhl HM, Ghosh M, Smith MK, et al. $\beta$-Catenin dosage is a critical determinant of tracheal basal cell fate determination. Am J Pathol 2011; 179: 367-379.

Giangreco A, Lu L, Vickers C, et al. $\beta$-Catenin determines upper airway progenitor cell fate and preinvasive squamous lung cancer progression by modulating epithelial-mesenchymal transition. J Pathol 2012; 226: 575-587.

Mahoney JE, Mori M, Szymaniak AD, et al. The Hippo pathway effector yap controls patterning and differentiation of airway epithelial progenitors. Dev Cell 2014; 30: 137-150.

Mcconnell AM, Yao C, Yeckes AR, et al. p53 regulates progenitor cell quiescence and differentiation in the airway. Cell Rep 2016; 17: 2173-2182.

Kumar PA, Hu Y, Yamamoto $\mathrm{Y}$, et al. Distal airway stem cells yield alveoli in vitro and during lung regeneration following H1N1 influenza infection. Cell 2011; 147: 525-538.

Vaughan AE, Brumwell AN, Xi Y, et al. Lineage-negative progenitors mobilize to regenerate lung epithelium after major injury. Nature 2015; 517: 621-625.

Zuo W, Zhang T, Wu DZA, et al. p63+Krt5+ distal airway stem cells are essential for lung regeneration. Nature 2015; 517: 616-620.

Pardo-Saganta A, Tata PR, Law BM, et al. Parent stem cells can serve as niches for their daughter cells. Nature 2015; 523: 597-601.

Gupta R, Radicioni G, Abdelwahab S, et al. Intercellular communication between airway epithelial cells is mediated by exosome-like vesicles. Am J Respir Cell Mol Biol 2019; 60: 209-220. 
Kadota T, Fujita Y, Araya J, et al. Human bronchial epithelial cell-derived extracellular vesicle therapy for pulmonary fibrosis via inhibition of TGF- $\beta$-WNT crosstalk. J Extracellular Vesicles 2021; 10: e12124.

$\mathrm{Xu} X$, Zheng L, Yuan Q, et al. Transforming growth factor- $\beta$ in stem cells and tissue homeostasis. Bone Research 2018; 6: 2.

Cho IJ, Lui PP, Obajdin J, et al. Mechanisms, hallmarks, and implications of stem cell quiescence. Stem Cell Rep 2019; 12: 1190-1200.

Flozak AS, Lam AP, Russell S, et al. $\beta$-Catenin/T-cell factor signaling is activated during lung injury and promotes the survival and migration of alveolar epithelial cells. J Biol Chem 2010; 285: 3157-3167.

Nabhan AN, Brownfield DG, Harbury PB, et al. Single-cell Wnt signaling niches maintain stemness of alveolar type 2 cells. Science 2018; 359: 1118-1123.

Barkauskas CE, Cronce MJ, Rackley CR, et al. Type 2 alveolar cells are stem cells in adult lung. J Clin Invest 2013; 123: 3025-3036.

Butler JP, Loring SH, Patz S, et al. Evidence for adult lung growth in humans. N Engl J Med 2012; 367: 244-247.

Desai TJ, Brownfield DG, Krasnow MA. Alveolar progenitor and stem cells in lung development, renewal and cancer. Nature 2014; 507: 190-194.

Zacharias WJ, Frank DB, Zepp JA, et al. Regeneration of the lung alveolus by an evolutionarily conserved epithelial progenitor. Nature 2018; 555: 251-255.

Ding BS, Nolan DJ, Guo P, et al. Endothelial-derived angiocrine signals induce and sustain regenerative lung alveolarization. Cell 2011; 147: 539-553.

Chung M-I, Bujnis M, Barkauskas CE, et al. Niche-mediated BMP/SMAD signaling regulates lung alveolar stem cell proliferation and differentiation. Development 2018; 145: dev163014.

Pociask DA, Scheller EV, Mandalapu S, et al. IL-22 is essential for lung epithelial repair following influenza infection. Am J Pathol 2013; 182: 1286-1296.

Quan Y, Wang Z, Gong L, et al. Exosome miR-371b-5p promotes proliferation of lung alveolar progenitor type II cells by using PTEN to orchestrate the PI3 K/Akt signaling. Stem Cell Res Ther 2017; 8: 138.

Mitchell A, Wanczyk H, Jensen T, et al. Human induced pluripotent stem cells ameliorate hyperoxia-induced lung injury in a mouse model. Am J Transl Res 2020; 12: 292-307.

Lee J-H, Tammela T, Hofree M, et al. Anatomically and functionally distinct lung mesenchymal populations marked by Lgr5 and Lgr6. Cell 2017; 170: 1149-1163.e1112.

Volckaert T, Dill E, Campbell A, et al. Parabronchial smooth muscle constitutes an airway epithelial stem cell niche in the mouse lung after injury. J Clin Investig 2011; 121: 4409-4419.

Zepp JA, Zacharias WJ, Frank DB, et al. Distinct mesenchymal lineages and niches promote epithelial self-renewal and myofibrogenesis in the lung. Cell 2017; 170: 1134-1148.e1110.

Han X, Wang R, Zhou Y, et al. Mapping the mouse cell atlas by microwell-seq. Cell 2018; 172: 1091-1107. e1017.

Volckaert T, Yuan T, Chao C-M, et al. Fgf10-Hippo epithelial-mesenchymal crosstalk maintains and recruits lung basal stem cells. Dev Cell 2017; 43: 48-59.e45.

Gupte VV, Ramasamy SK, Reddy R, et al. Overexpression of fibroblast growth factor-10 during both inflammatory and fibrotic phases attenuates bleomycin-induced pulmonary fibrosis in mice. Am J Respir Crit Care Med 2009; 180: 424-436.

Sirianni FE, Chu FS, Walker DC. Human alveolar wall fibroblasts directly link epithelial type 2 cells to capillary endothelium. Am J Respir Crit Care Med 2003; 168: 1532-1537.

Green $\mathrm{J}$, Endale $\mathrm{M}$, Auer $\mathrm{H}$, et al. Diversity of interstitial lung fibroblasts is regulated by platelet-derived growth factor receptor $\alpha$ kinase activity. Am J Respir Cell Mol Biol 2016; 54: 532-545.

Haj-Salem I, Plante S, Gounni AS, et al. Fibroblast-derived exosomes promote epithelial cell proliferation through TGF- $\beta 2$ signalling pathway in severe asthma. Allergy 2018; 73: 178-186.

Kadota T, Yoshioka Y, Fujita Y, et al. Extracellular vesicles from fibroblasts induce epithelial-cell senescence in pulmonary fibrosis. Am J Respir Cell Mol Biol 2020; 63: 623-636.

Barkauskas CE, Chung M-I, Fioret B, et al. Lung organoids: current uses and future promise. Development 2017; 144: 986-997.

Zysman M, Baptista BR, Essari L-A, et al. Targeting p16INK4a promotes lipofibroblasts and alveolar regeneration after early-life injury. Am J Respir Crit Care Med 2020; 202: 1088-1104.

Lacy SH, Woeller CF, Thatcher TH, et al. Activated human lung fibroblasts produce extracellular vesicles with antifibrotic prostaglandins. Am J Respir Cell Mol Biol 2019; 60: 269-278.

Byrne AJ, Mathie SA, Gregory LG, et al. Pulmonary macrophages: key players in the innate defence of the airways. Thorax 2015; 70: 1189-1196.

Iwasaki A, Foxman EF, Molony RD. Early local immune defences in the respiratory tract. Nat Rev Immunol 2017; 17: 7-20.

Lechner AJ, Driver IH, Lee J, et al. Recruited monocytes and type 2 immunity promote lung regeneration following pneumonectomy. Cell Stem Cell 2017; 21: 120-134.e127. 
Hung L-Y, Sen D, Oniskey TK, et al. Macrophages promote epithelial proliferation following infectious and non-infectious lung injury through a Trefoil factor 2-dependent mechanism. Mucosal Immunol 2019; 12: 64-76.

Katsura H, Kobayashi Y, Tata PR, et al. IL-1 and TNF $\alpha$ contribute to the inflammatory Niche to enhance alveolar regeneration. Stem Cell Rep 2019; 12: 657-666.

Tadokoro T, Wang Y, Barak LS, et al. IL-6/STAT3 promotes regeneration of airway ciliated cells from basal stem cells. Proc Natl Acad Sci 2014; 111: E3641-E3649.

Kulshreshtha A, Ahmad T, Agrawal A, et al. Proinflammatory role of epithelial cell-derived exosomes in allergic airway inflammation. J Allergy Clin Immunol 2013; 131: 1194-1203.e1114.

Bourdonnay E, Zaslona Z, Penke LR, et al. Transcellular delivery of vesicular SOCS proteins from macrophages to epithelial cells blunts inflammatory signaling. J Exp Med 2015; 212: 729-742.

Yoshimura A, Naka T, Kubo M. SOCS proteins, cytokine signalling and immune regulation. Nat Rev Immunol 2007; 7: 454-465.

Draijer C, Speth JM, Penke LRK, et al. Resident alveolar macrophage-derived vesicular SOCS3 dampens allergic airway inflammation. FASEB J 2020; 34: 4718-4731.

Zhu Z, Zhang D, Lee $\mathrm{H}$, et al. Macrophage-derived apoptotic bodies promote the proliferation of the recipient cells via shuttling microRNA-221/222. J Leukoc Biol 2017; 101: 1349-1359.

Guiot J, Cambier M, Boeckx A, et al. Macrophage-derived exosomes attenuate fibrosis in airway epithelial cells through delivery of antifibrotic miR-142-3p. Thorax 2020; 75: 870-881.

Pittenger MF. Multilineage potential of adult human mesenchymal stem cells. Science 1999; 284: 143-147.

Wang L, Shi M, Tong L, et al. Lung-resident mesenchymal stem cells promote repair of LPS-induced acute lung injury via regulating the balance of regulatory T cells and Th17 cells. Inflammation 2019; 42: 199-210.

Lee C, Mitsialis SA, Aslam M, et al. Exosomes mediate the cytoprotective action of mesenchymal stromal cells on hypoxia-induced pulmonary hypertension. Circulation 2012; 126: 2601-2611.

Fujita Y, Kadota T, Araya J, et al. Clinical application of mesenchymal stem cell-derived extracellular vesicle-based therapeutics for inflammatory lung diseases. J Clin Med 2018; 7: 355.

Khatri M, Richardson LA, Meulia T. Mesenchymal stem cell-derived extracellular vesicles attenuate influenza virus-induced acute lung injury in a pig model. Stem Cell Res Ther 2018; 9: 17.

Tang X-D, Shi L, Monsel A, et al. Mesenchymal stem cell microvesicles attenuate acute lung injury in mice partly mediated by Ang-1 mRNA. Stem Cells 2017; 35: 1849-1859.

Monsel A, Zhu Y-G, Gennai S, et al. Therapeutic effects of human mesenchymal stem cell-derived microvesicles in severe pneumonia in mice. Am J Respir Crit Care Med 2015; 192: 324-336.

Zhu Y-G, Feng X-M, Abbott J, et al. Human mesenchymal stem cell microvesicles for treatment of Escherichia coli endotoxin-induced acute lung injury in mice. Stem Cells 2014; 32: 116-125.

Silva JD, Su Y, Calfee CS, et al. Mesenchymal stromal cell extracellular vesicles rescue mitochondria dysfunction and improve barrier integrity in clinically relevant models of ARDS. Eur Respir J 2021; 58: 2002978.

Islam MN, Das SR, Emin MT, et al. Mitochondrial transfer from bone-marrow-derived stromal cells to pulmonary alveoli protects against acute lung injury. Nat Med 2012; 18: 759-765.

Yi X, Wei X, Lv H, et al. Exosomes derived from microRNA-30b-3p-overexpressing mesenchymal stem cells protect against lipopolysaccharide-induced acute lung injury by inhibiting SAA3. Exp Cell Res 2019; 383: 111454.

Li JW, Wei L, Han Z, et al. Mesenchymal stromal cells-derived exosomes alleviate ischemia/reperfusion injury in mouse lung by transporting anti-apoptotic miR-21-5p. Eur J Pharmacol 2019; 852: 68-76.

Wei X, Yi X, Lv H, et al. MicroRNA-377-3p released by mesenchymal stem cell exosomes ameliorates lipopolysaccharide-induced acute lung injury by targeting RPTOR to induce autophagy. Cell Death Dis 2020; 11: 657.

De Jong OG, Verhaar MC, Chen Y, et al. Cellular stress conditions are reflected in the protein and RNA content of endothelial cell-derived exosomes. J Extracell Vesicles 2012; 1: 18396.

Pardo A, Selman M. The interplay of the genetic architecture, aging, and environmental factors in the pathogenesis of idiopathic pulmonary fibrosis. Am J Respir Cell Mol Biol 2021; 64: 163-172.

Martin-Medina A, Lehmann M, Burgy O, et al. Increased extracellular vesicles mediate WNT5A signaling in idiopathic pulmonary fibrosis. Am J Respir Crit Care Med 2018; 198: 1527-1538.

Chanda D, Otoupalova E, Hough KP, et al. Fibronectin on the surface of extracellular vesicles mediates fibroblast invasion. Am J Respir Cell Mol Biol 2019; 60: 279-288.

2 Fujita Y, Araya J, Ito S, et al. Suppression of autophagy by extracellular vesicles promotes myofibroblast differentiation in COPD pathogenesis. J Extracell Vesicles 2015; 4: 28388.

03 Genschmer KR, Russell DW, Lal C, et al. Activated PMN exosomes: pathogenic entities causing matrix destruction and disease in the lung. Cell 2019; 176: 113-126.e115.

Soni S, Garner JL, O'Dea KP, et al. Intra-alveolar neutrophil-derived microvesicles are associated with disease severity in COPD. Am J Physiol Lung Cell Mol Physiol 2021; 320: L73-L83. 
105 Chambers DC, Enever D, llic N, et al. A phase 1b study of placenta-derived mesenchymal stromal cells in patients with idiopathic pulmonary fibrosis. Respirology 2014; 19: 1013-1018.

106 Glassberg MK, Minkiewicz J, Toonkel RL, et al. Allogeneic human mesenchymal stem cells in patients with idiopathic pulmonary fibrosis via intravenous delivery (AETHER). Chest 2017; 151: 971-981.

107 Serrano-Mollar A, Nacher M, Gay-Jordi G, et al. Intratracheal transplantation of alveolar type II cells reverses bleomycin-induced lung fibrosis. Am J Respir Crit Care Med 2007; 176: 1261-1268.

108 Serrano-Mollar A, Gay-Jordi G, Guillamat-Prats R, et al. Safety and tolerability of alveolar type II cell transplantation in idiopathic pulmonary fibrosis. Chest 2016; 150: 533-543.

109 Vizoso F, Eiro N, Cid S, et al. Mesenchymal stem cell secretome: toward cell-free therapeutic strategies in regenerative medicine. Int J Mol Sci 2017; 18: 1852.

110 Menasche P. Cell therapy trials for heart regeneration - lessons learned and future directions. Nat Rev Cardiol 2018; 15: 659-671.

111 Zhou Y, Yamamoto Y, Xiao Z, et al. The immunomodulatory functions of mesenchymal stromal/stem cells mediated via paracrine activity. J Clin Med 2019; 8: 1025.

112 Lukomska B, Stanaszek L, Zuba-Surma E, et al. Challenges and controversies in human mesenchymal stem cell therapy. Stem Cells Int 2019; 2019: 9628536.

113 Willis GR, Fernandez-Gonzalez A, Reis M, et al. Mesenchymal stromal cell-derived small extracellular vesicles restore lung architecture and improve exercise capacity in a model of neonatal hyperoxia-induced lung injury. J Extracell Vesicles 2020; 9: 1790874.

114 Dinh P-UC, Paudel D, Brochu H, et al. Inhalation of lung spheroid cell secretome and exosomes promotes lung repair in pulmonary fibrosis. Nat Commun 2020; 11: 1064.

115 Zhou Y, Li P, Goodwin AJ, et al. Exosomes from endothelial progenitor cells improve outcomes of the lipopolysaccharide-induced acute lung injury. Critical Care 2019; 23: 44.

116 Khalaj K, Figueira RL, Antounians L, et al. Systematic review of extracellular vesicle-based treatments for lung injury: are EVs a potential therapy for COVID-19? J Extracell Vesicles 2020; 9: 1795365.

117 Balasubramaniam V, Ryan SL, Seedorf GJ, et al. Bone marrow-derived angiogenic cells restore lung alveolar and vascular structure after neonatal hyperoxia in infant mice. Am J Physiol Lung Cell Mol Physiol 2010; 298: L315-L323.

118 Takahashi K, Yamanaka S. Induction of pluripotent stem cells from mouse embryonic and adult fibroblast cultures by defined factors. Cell 2006; 126: 663-676.

119 Takahashi K, Tanabe K, Ohnuki M, et al. Induction of pluripotent stem cells from adult human fibroblasts by defined factors. Cell 2007; 131: 861-872.

120 Gazdhar A, Ravikumar P, Pastor J, et al. Alpha-Klotho enrichment in induced pluripotent stem cell secretome contributes to antioxidative protection in acute lung injury. Stem Cells 2018; 36: 616-625.

121 Gazdhar A, Grad I, Tamò L, et al. The secretome of induced pluripotent stem cells reduces lung fibrosis in part by hepatocyte growth factor. Stem Cell Res Ther 2014; 5: 123.

122 Dane DM, Cao K, Zhang YA, et al. Inhalational delivery of induced pluripotent stem cell secretome improves postpneumonectomy lung structure and function. J Appl Physiol (1985) 2020; 129: 1051-1061.

123 Katsura H, Sontake V, Tata A, et al. Human lung stem cell-based alveolospheres provide insights into SARS-CoV-2-mediated interferon responses and pneumocyte dysfunction. Cell Stem Cell 2020; 27: 890-904. e898.

124 Youk J, Kim T, Evans KV, et al. Three-dimensional human alveolar stem cell culture models reveal infection response to SARS-CoV-2. Cell Stem Cell 2020; 27: 905-919.e910. 